\title{
El desarrollo docente del formador de profesores: una propuesta orientada hacia el análisis de incidentes críticos auténticos*
}

The teaching development of teachers' educators: a proposal for the analysis to real critical incidents

\section{O desenvolvimento do professor formador de professores: uma proposta orientada para a análise de incidentes críticos legítimos}

\section{Claudia Contreras $C$.}

Universidad Austral de Chile.

Telf.: (562)222931. Correo electrónico: claudia.contreras@uach.cl

\begin{abstract}
RESUMEN
Esta investigación explora en la identidad del profesorado universitario de magisterio y en su transformación como objeto último del desarrollo docente. Específicamente, se analizó cómo se desarrolla la identidad de los profesores en sus escenarios institucionales y los incidentes críticos (IC) ocurridos en las aulas universitarias, ofreciendo una profundización que permitió realizar una propuesta de formación permanente que fue diseñada, implementada y evaluada. Entre las conclusiones se destaca la concepción de una identidad del profesorado universitario como modelo de integración entre teoría y práctica pedagógica. Junto con lo anterior, los IC identificados se asocian principalmente a tres situaciones: las evaluaciones del aprendizaje, los déficits en las competencias de los estudiantes y la introducción de cambios en las normas o metodologías de aula. Adicionalmente, entre los profesores participantes de la formación identificamos un cambio en la concepción de IC, de él se rescata su utilidad para el desarrollo docente y el análisis reflexivo.
\end{abstract}

Palabras clave: formador de profesores, desarrollo docente, incidentes críticos.

\begin{abstract}
This research explores the identity of university teachers of teaching professions, and their transformation as ultimate object of the teacher development. Specifically, it was discussed how the identity of the teachers is developed in their institutional settings and the critical incidents (CI) occurred in university classrooms, offering an analysis that allowed the development of a proposal for continuing education, which was designed, implemented and evaluated. Among the conclusions it is highlighted an identity conception of university teachers as model of integration between pedagogical theory and practice. Along with the above, the ICs identified are mainly associated with three situations, learning assessments, deficits in the skills of the students, and the introduction of changes in the rules or methodologies classroom. Additionally, between teachers training participants, we identify a change in the design of IC, highlighting its utility for the teacher development and reflective analysis.
\end{abstract}

Key words: teacher educator, teacher development, critical incidents.

\footnotetext{
* Este estudio forma parte de la tesis doctoral "La formación del profesorado universitario orientada hacia la transformación de la identidad docente. Una propuesta basada en incidentes críticos". Desarrollada en el marco de una Beca Chile doctorado en el extranjero, obtenida por la autora.
} 


\section{RESUMO}

Explora-se a identidade do professorado universitário do Magistério e sua transformação como objeto último do desenvolvimento docente. Especificamente, analisou-se como se desenvolve a identidade dos professores em seus cenários institucionais e os incidentes críticos (IC) ocorridos nas aulas universitárias, oferecendo um aprofundamento que permitiu uma proposta de formação continuada de professores, a qual foi pensada, implementada e avaliada. Entre as conclusões, destaca-se a concepção de uma identidade do professorado universitário como modelo de integração entre teoria e prática pedagógica. Junto do exposto, os ICs identificados se associaram principalmente a três situações: as avaliações da aprendizagem, os déficits das competências dos estudantes e a introdução de transformações das normas ou metodologias de ensino. Também, identifica-se uma transformação na concepção de IC entre os professores participantes da formação, resgatando sua utilidade para o desenvolvimento docente e para a análise reflexiva.

Palavras-chave: formador de professores, desenvolvimento docente, incidentes críticos.

\section{INTRODUCCIÓN}

En la actualidad, el mundo evoluciona rápidamente afectando distintos ámbitos de la sociedad, es sabido que entre los espacios que mayormente se han visto influenciados están las aulas universitarias, su alumnado y la docencia. En este sentido, hoy en día la enseñanza universitaria debe implementar nuevas maneras de educar y abordar las relaciones que se establecen dentro del aula, pensar en el desarrollo y la innovación, potenciar habilidades cognitivas, sociales, afectivas y comunicativas de sus alumnos, hacerse cargo de formar a sujetos participativos, creativos, proactivos, etc., con el fin de responder a las expectativas que la sociedad ha formulado, a partir de una nueva realidad más compleja y globalizada (Hargreaves, 2005; Zabalza, 2009).

En ese sentido, si podemos identificar las necesidades y los resultados a los que se aspira llegar en la educación universitaria, nos queda pendiente el desafío de investigar qué sucede en la búsqueda y consecución de los retos propuestos, cómo logramos una docencia que efectivamente responda a los cambios y demandas permanentes, cómo llegamos a una enseñanza abierta a la incertidumbre sin desorientarnos. El panorama que se abre en medio de estos cuestionamientos se problematiza en la medida en que reconocemos el escenario, los actores y la meta, pero tenemos en frente un horizonte confuso.

Como sabemos, la calidad de un programa formativo depende fundamentalmente de las condiciones de enseñanza a las que se ven expuestos los futuros profesionales, derivándose de esto que las habilidades e intervenciones pedagógicas de los profesores cobren especial relevancia. Lo mencionado anteriormente resulta de especial importancia, considerando que muchos profesores deben formar en un contexto complejo, sin necesariamente contar con los recursos y la formación suficiente para enfrentar los desafíos de la docencia universitaria actual. En consecuencia, cuando nos proponemos investigar e intervenir en el ámbito del desarrollo profesional docente en la universidad, nos referimos a que debemos cambiar un enfoque de formación continua más bien rígido, aislado y homogéneo, para orientarnos a consolidar espacios de construcción colegiados, diversos y que atiendan a problemas auténticos, implicando con ello un punto de inflexión, una transformación del profesor, un giro cultural hacia la colaboración y una observación sistemática de la realidad educativa en la universidad.

Finalmente, podemos señalar que el propósito último de esta investigación se orienta hacia la formación del profesorado universitario de pedagogía, utilizando los incidentes críticos auténticos como mediadores de análisis para la transformación identitaria. 


\section{EL DESARROLLO DOCENTE DEL FORMADOR DE PROFESORES}

Hasta hace un tiempo, y desde una lógica acumulativa, se entendía que el docente universitario en su rol de enseñante debía ser un repositorio y transmisor de conocimiento. Sin embargo, con la influencia de los cambios sociales, el desarrollo de nuevas teorías epistemológicas y del aprendizaje, esta forma de comprender el trabajo docente se ha ido transformando, añadiéndose otras miradas que complejizan y amplían el quehacer del profesor en la universidad (Briggs, 2005; De la Cruz, 2003).

Avanzando en esta línea, podemos señalar que actualmente el profesor universitario, junto con ser un experto en su disciplina, un investigador y un gestor, debe saber enseñar, lo cual implica facilitar las condiciones de aprendizaje, pensando en las características del estudiantado de hoy y el perfil profesional que se le demanda formar. En relación a esta caracterización, algunos autores señalan que el docente universitario además debe dominar algunos temas genéricos relacionados con el enseñar, como la ética -o los valores que desea promover en sus alumnos-, la reflexión, la innovación y el aprendizaje permanente; además de las habilidades sociales requeridas para relacionarse con una diversidad de alumnos, colegas y autoridades académicas (Badley, 2000; Tedesco y Tenti, 2002).

Siguiendo lo planteado anteriormente, podemos señalar que la formación permanente del profesorado universitario debe responder a fenómenos continuos, dinámicos y multidimensionales, donde las tareas sean contextualizadas desde una visión cualitativa y de proceso, es decir, a partir del conocimiento condicional o estratégico, que se deriva de una relación ajustada al entorno, fundamentada en la realidad y la teoría (Badia y Monereo, 2011; Monereo et al., 2009). En este sentido, es importante que la formación permanente no sólo asegure espacios de aprendizaje de forma tradicional, sino también ambientes diversificados, donde el trabajo permita una revisión de las representaciones del profesor (creencias, conceptos, roles, sentimientos, etc.), en pos de trabajar sobre una redescripción representacional de la realidad (es) para desaprender y aprender en el ejercicio profesional atendiendo al contexto (Karmiloffñ-Smith, 1994).

En coherencia con lo anterior, dejar atrás la tradición seguida en el desarrollo profesional docente implica que debemos cambiar el enfoque de formación permanente, para orientarnos a consolidar espacios de aprendizaje que repercutan en las posibilidades de éxito de los enseñantes y los aprendices, en determinado escenario formativo.

Es entonces que, considerando lo previamente descrito, nos preguntamos ¿Hacia dónde focalizar el desarrollo profesional del docente universitario?, ¿Cómo encauzar todos los dilemas, demandas y problemas en un proceso tan complejo?. Sin la intención de buscar respuestas acabadas, encontramos aportes que resaltan algunos aspectos, como lo señalado por De la Cruz (2003), en relación a que la formación continua del profesorado universitario debe ser adaptada al contexto y desde una perspectiva crítica, que colabore en que el docente pueda cambiar y mejorar el sistema, más que adaptarse a él. En complemento, Guskey (2000) enfatiza que debe darse una discusión previa y también recurrente a la formación, que permita clarificar los intereses y objetivos de ésta, así como un camino para alcanzarlos, señalando que sin lo anterior como premisa, resultará difícil mejorar la calidad del desarrollo profesional docente. Desde esta óptica, una consideración importante en el contexto de la educación superior es que por lo general el docente se ha formado en una disciplina, y a partir de ella se ha involucrado en la tarea de enseñar, extrayendo desde su experiencia, herramientas para diseñar programas, desarrollar una clase y relacionarse 
Estudios Pedagógicos, vol. XL, Número Especial 1: 49-69, 2014

EL DESARROLLO DOCENTE DEL FORMADOR DE PROFESORES: UNA PROPUESTA ORIENTADA HACIA EL

ANÁLISIS DE INCIDENTES CRÍTICOS AUTÉNTICOS

con sus alumnos, para hacer de su docencia una vivencia gratificante y efectiva, lo cual, como ya se ha mencionado, será insuficiente para enfrentar los desafíos que actualmente encontrará en el aula.

En resumen, si bien es cierto que frente a este tema se abren muchas aristas, por el momento podemos reflexionar en torno a tres ejes problemáticos que cruzan la docencia universitaria hoy en día:

- La carrera del profesor universitario se traduce principalmente en dos ámbitos de difícil encuentro, la investigación y la enseñanza; claramente se ha establecido que una actividad debe nutrirse de la otra, no obstante se ha tendido a ignorar la profesionalidad docente como un quehacer distinto, que debe integrarse a otras experticias, teniendo métodos y demandas particulares, propias del enseñar.

- El profesor debe ir evolucionando y mejorando su docencia para atender a su alumnado con variadas estrategias de enseñanza y recursos didáctico-pedagógicos, en razón a que cada día la población estudiantil universitaria es más numerosa y heterogénea. Éstas, entre otras razones, hacen que su propia experiencia y formación inicial sean insuficientes para atender a las necesidades de aprendizaje de sus estudiantes.

- Las actividades propias de investigar y enseñar se suman a la gestión tanto de la docencia como de otros aspectos administrativos vinculados a la enseñanza y la búsqueda de financiación para proyectos de investigación o extensión.

Cabe señalar que al revisar la literatura experimentamos cierto grado de incertidumbre respecto de si estos ejes - problemas- están recibiendo la atención que merecen en la actualidad. En este sentido, podemos afirmar que la enseñanza universitaria, como ámbito para intervenir y estudiar, es, de alguna manera, de tardía discusión entre las investigaciones y la literatura especializada (Cornejo, 2007; Zabalza, 2009).

Básicamente, a partir de las premisas y reflexiones presentadas argumentamos la necesidad de encontrar el equilibrio entre una formación del profesorado centrada en la reflexión, que focaliza el aprendizaje docente en procesos intrapsicológicos y, por otro lado, una formación basada en técnicas y métodos que focalizan el aprendizaje docente en un cúmulo de procedimientos por hacer.

\section{LA IDENTIDAD DOCENTE EN TIEMPOS DE CAMBIO}

Desde hace un tiempo, distintos autores han reconocido que la identidad profesional del docente se construye y reconstruye en un espacio y en un tiempo determinado, en donde el sujeto se nutre y aporta en el entorno (Day, 2006; Galaz, 2011). En este espacio, desde un marco de historicidad en que la acción se ubica en la actualidad desde un pasado que es evocado y traído al presente, en sincronía con una imagen futura respecto de quien se quiere ser, es que la identidad se desarrolla (Monereo y Badia, 2011). Es aquí entonces cuando creemos necesario integrar una característica de la identidad como compromiso futuro, una visión imaginada de sí mismo que además tiene un reflejo en la actividad, proyectada en el quehacer docente, que es más que un discurso.

Al profundizar en los componentes de la identidad docente, podemos definir tres aspectos identitarios en constante interacción y que resultan relevantes para su 
conceptualización: a) las concepciones personales sobre distintos aspectos de la docencia; b) los procedimientos y estrategias para la enseñanza; c) los sentimientos vinculados al desempeño como profesor. A nuestro juicio, estos componentes tienen una relación directa con la representación del mundo que tiene el docente, de lo que se quiere ser y hacer en su profesión, lo que vivencia y la forma en que sus acciones se ajustan en su entorno (Badia, Monereo y Meneses, 2011). A continuación ofrecemos una descripción de cada uno de estos componentes:

Concepciones personales: constituyen las distintas representaciones que el profesor desarrolla acerca de lo que es enseñar, aprender y evaluar, así como también sobre el rol o roles que asume en el ámbito universitario. Estas concepciones, en forma de conocimientos, creencias o teorías personales explícitas o implícitas, conforman la estructura conceptual de la identidad, en tanto que le permiten interpretar, dar sentido y cierta coherencia a su relación con el entorno laboral (Monereo y Badia, 2011; Pozo, 2006).

Procedimientos y estrategias docentes: asumimos con esto que el profesor desarrolla ciertas representaciones sobre lo que hace en el aula para alcanzar los aprendizajes con sus estudiantes, las expectativas sobre su desempeño y los propósitos que se deriven de su actuación. Estas representaciones involucran las maneras en que enseña, evalúa y gestiona conflictos, entre otras; es decir, definiendo de esta forma su actuar de manera más o menos deliberada y reflejada en el discurso (Monereo et al., 2009; Badia, Monereo y Meneses, 2011).

Sentimientos vinculados al desempeño: nos referimos a las representaciones afectivas que pueden abarcar los propios sentimientos del profesor, así como también la interpretación de las emociones o sensaciones vinculadas al trabajo docente. Estas representaciones pueden tener una connotación positiva (como satisfacción, compromiso, orgullo, etc.) o negativa (como frustración, culpa, ansiedad, etc.), en tanto que dependerán de las asociaciones que el profesor establezca con su trabajo en general o con episodios significativos en particular, como por ejemplo los IC que sucedan (Meijers, 2003; Navarro et al., 2012).

En adición a lo anterior, cuando señalamos estos tres aspectos como contenidos de la identidad, también hacemos referencia a que en su desarrollo se intersectan y movilizan en los posicionamientos identitarios, sin necesariamente guardar una coherencia irrestricta entre los tres componentes, puesto que asumidos de manera flexible, estos tres pilares igualmente pueden entrar en contradicción (en el posicionamiento), conflicto o negociación, dependiendo de qué resulte dominante o adaptativo entre las concepciones, sentimientos y procedimientos desarrollados por el docente y lo que está demandando el contexto inmediato.Desde el reconocimiento de que la identidad docente es un proceso complejo, amplio, dinámico y multidimensional, asumimos la premisa de que sólo a través de la comprensión del sujeto integral lograremos encontrar alternativas de formación que puedan impactar y perdurar. En consecuencia, resultará más adecuado referirnos a la transformación de la identidad docente, más que a un fortalecimiento o cambio de ella, cuando nos situamos en el marco de la formación del profesorado.

En síntesis, en el caso de los docentes universitarios, la identidad profesional como constructo debe ser comprendida como un proceso de interacción e integración de perturbaciones y confirmaciones. A partir de lo anteriormente expuesto será de suma importancia considerar que el desarrollo docente tenga como propósito un cambio más profundo en el profesional, que implique la transformación de la identidad como objetivo último (Akkerman y Meijer, 2010; Day, 2005). 
Estudios Pedagógicos, vol. XL, Número Especial 1: 49-69, 2014

EL DESARROLLO DOCENTE DEL FORMADOR DE PROFESORES: UNA PROPUESTA ORIENTADA HACIA EL ANÁLISIS DE INCIDENTES CRÍTICOS AUTÉNTICOS

\section{LOS INCIDENTES CRÍTICOS COMO ESTRATEGIA DE FORMACIÓN DOCENTE}

Tal como en otros espacios sociales, en las salas de clases universitarias ocurren situaciones inesperadas para las cuales, en principio, no hay respuestas aseguradas. Al realizar sus clases, un profesor enfrenta muchas veces un horizonte abierto que implica poner en práctica sus competencias para dar respuesta a demandas que con alguna frecuencia no ha podido anticipar. Adicionalmente, si los espacios formativos que le permitan canalizar inquietudes son escasos o poco adecuados, será difícil que podamos problematizar y aumentar las posibilidades de una actuación resolutiva y estratégica en el profesorado. En la medida que las situaciones inesperadas se nos vuelven difíciles, no las resolvemos o las enfrentamos de manera desajustada, se convierten en un problema, perturbando el quehacer, sentir y pensar profesional. Es entonces que, a partir de estas situaciones imprevistas se desprende una sensación de inestabilidad o perturbación que nos sitúa dentro de un evento llamado incidente crítico.

En términos generales, un incidente nos viene a señalar algo que acontece en el transcurso de un proceso y cambia de alguna manera la dirección que tenía prevista. Lo crítico, en tanto, estaría dado porque al interrumpir lo presupuestado, quienes interpretamos o vivimos un incidente hacemos eco de su ocurrencia experimentando una crisis que, en el caso de la docencia, dificulta o impide una salida satisfactoria en una actividad de enseñanza y aprendizaje (Monereo y Monte, 2011; Tripp, 1993).

Al analizar este concepto, y en línea con nuestra investigación, se comprenderá un IC como un evento de ocurrencia inesperada que sucede en el marco del trabajo docente y que pone en cuestión algún aspecto que el profesor no ha anticipado y que, al significarse como negativo, supera cierto umbral emocional poniendo al profesor en conflicto (subjetivo o intersubjetivo). De este modo se espera que para sobreponerse, recuperarse y continuar con su tarea, al profesor no le sea suficiente aferrarse a una estrategia local, sino que necesitará utilizar mecanismos psicológicos de re-estructuración cognitiva que configuren una nueva o nuevas versiones de sí mismo (Contreras, Monereo y Badia, 2010; Monereo et al., 2009).

Por otra parte, tal como establecen algunos autores (Monereo y Monte, 2011), el uso de incidentes críticos en la formación del profesorado ofrece algunas posibilidades en tanto que:

- Explicitan problemas genuinos en el ejercicio docente, los cuales muchas veces permanecen latentes o son vivenciados desde lo implícito. De esta manera ayudan a representar externamente los problemas que ocurren en el aula y a trabajar con creencias, concepciones, actitudes, sentimientos y actuaciones implicados en la docencia.

- Son un punto de partida para la discusión interpersonal en la que se entrelazan distintos aspectos del ejercicio profesional (pedagógico, disciplinar, entre otros), facilitando además el acceso al trabajo con la teoría y la práctica de manera integrada.

- Exploran el despliegue de competencias para la resolución de problemas auténticos y la toma de decisiones en la docencia.

- Rescatan el conocimiento profesional desde la práctica (enfoque reflexivo), reconociendo saberes en la experiencia de los propios docentes, en lo individual y colectivo, para una actuación estratégica.

Es así como, a partir de los puntos anteriormente descritos, comprendemos el IC como un medio y una posibilidad de acercamiento a las dificultades genuinas que enfrentan los docentes en las aulas. 
Reforzando lo anterior, podemos señalar que, tal como en otros espacios sociales, en las salas de clases universitarias ocurren situaciones inesperadas para las cuales, en principio, no hay respuestas aseguradas, por lo cual resulta relevante abrir posibilidades en la investigación de los IC y su uso en la formación docente. En este mismo sentido, los IC se ofrecen como un primer encuadre que facilita una mirada más amplia y compleja de los problemas que enfrentan los docentes en la enseñanza, condición fundamental para un trabajo auténtico y los cambios que sea necesario llevar a cabo para la realización de una docencia efectiva (Contreras, Monereo y Badia, 2010).

\section{METODOLOGÍA}

\subsection{DISEÑO Y OBJETIVOS}

El presente estudio se ha planteado desde un enfoque cualitativo, de corte descriptivointerpretativo, desarrollando un proceso investigativo de intercambio y construcción compartido entre la investigadora y los participantes, asegurando la correspondencia entre la complejidad de la realidad estudiada y los sujetos involucrados, con una perspectiva flexible y comprensiva (Hernández, Fernández y Baptista, 2010). En esta misma línea, trabajamos particularmente en el marco del "Estudio de casos" como estrategia de profundización, dado que nos ofrecía una aproximación a la realidad que debía ser conocida e interpretada, y al mismo tiempo facilitaba un acercamiento empático y no impositivo.

El objetivo general que nos hemos propuesto se resume en: valorar el impacto de un programa de formación para el profesorado universitario, basado en el trabajo con incidentes críticos como medio para la transformación de la identidad profesional docente. Nos planteamos alcanzar este objetivo a partir del trabajo desarrollado en torno a los siguientes objetivos específicos:

- Analizar los componentes de la identidad profesional docente, a partir del discurso de los profesores, en relación a su experiencia como profesores en la formación inicial de maestros.

- Caracterizar los incidentes críticos que han afrontado los profesores universitarios en su experiencia como formadores en magisterio.

- Diseñar un programa de actividades teóricas y empíricamente fundamentadas para la formación docente del profesorado universitario de magisterio, basado en incidentes críticos.

- Evaluar las consecuencias de un programa de actividades para la formación docente del profesorado universitario de magisterio a partir de incidentes críticos.

\subsection{PARTICIPANTES}

En la primera fase participaron 20 docentes que trabajaban en tres facultades de educación en distintas universidades, cada uno de ellos fue entrevistado a partir de un guion. En la segunda fase participaron 6 docentes, que formaron parte del grupo inicial, quienes realizaban clases en la misma facultad de educación de una universidad tradicional de carácter complejo. De este último grupo, 5 docentes tuvieron una participación estable en las actividades de formación, de manera tal que formaron parte de la tercera fase del estudio (de evaluación), en la que fueron entrevistados en diferentes oportunidades, de forma individual y grupal. 
Estudios Pedagógicos, vol. XL, Número Especial 1: 49-69, 2014

EL DESARROLLO DOCENTE DEL FORMADOR DE PROFESORES: UNA PROPUESTA ORIENTADA HACIA EL

ANÁLISIS DE INCIDENTES CRÍTICOS AUTÉNTICOS

La figura que a continuación se presenta resume participantes y fases de desarrollo de la investigación:

Figura 1. Participantes y fases de desarrollo de la investigación

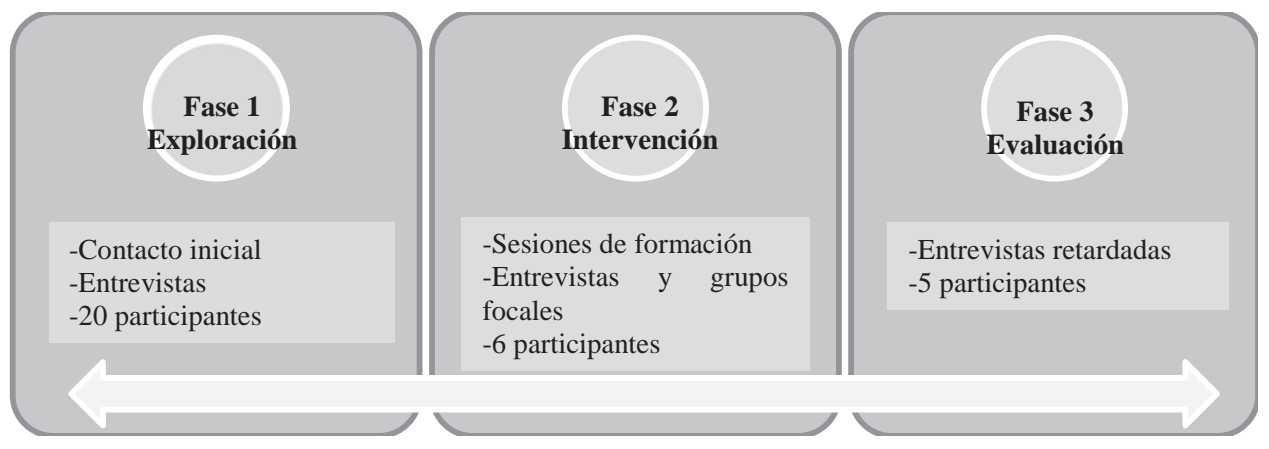

\subsection{REGISTRO Y ANÁLISIS}

Los instrumentos de recogida de información apuntaron primero a explorar y luego a profundizar en las narraciones de los participantes, en tanto se traducían en descripciones, interpretaciones, reflexiones, cuestionamientos y evaluaciones respecto a su identidad profesional, los incidentes críticos experimentados y su participación en las actividades de formación, en paralelo al desarrollo de las fases contempladas en este estudio. De manera concreta, recurrimos principalmente a tres técnicas de recolección de datos que utilizamos en las distintas fases del proceso, ellas nos proporcionaron la retroalimentación y datos requeridos. La Tabla 1 resume la utilización o aplicación de instrumentos:

Tabla 1. Inventario de instrumentos/técnicas para el registro de datos

\begin{tabular}{|lcccc|}
\hline Instrumento & Fase 1 & Fase 2 & Fase 3 & Total \\
\hline Entrevistas S-E & 20 & 14 & 5 & 27 \\
\hline Pautas de análisis & 0 & 17 & 0 & 17 \\
\hline Grupos focales & 0 & 4 & 0 & 4 \\
\hline
\end{tabular}

Fuente. Elaboración propia.

Por otra parte, las unidades de análisis fueron construidas a partir de los siguientes temas: a) concepciones sobre enseñanza, aprendizaje, roles y principios educativos, estrategias docentes y sentimientos acerca de la experiencia como profesor universitario; b) elementos relativos a incidentes críticos que habían ocurrido en clases con futuros maestros frente a escenarios pasados, actuales e incluso supuestos; c) otros aspectos referidos a la experiencia durante el proceso de formación (fase 2).

Para el tratamiento de los datos y la elaboración de los resultados adoptamos la Grounded Theory como enfoque de análisis (Strauss y Corbin, 2002). Esta perspectiva nos facilitó una revisión amplia y profunda de la información que fuimos elaborando, con la flexibilidad que ameritaba la investigación. En coherencia con este enfoque, nos 
planteamos una vía de codificación que permitió el microanálisis de los datos, la generación de categorías y la combinación de procesos de codificación abierta, axial y selectiva.

Por otra parte, antes de comenzar la primera fase de recogida de datos, sometimos a evaluación el guion de la entrevista inicial que realizaríamos. Esta evaluación buscó llegar a acuerdos a partir del análisis de cada pregunta y la dimensión sobre la cual nos proponíamos recoger datos. En este procedimiento participaron 3 jueces y consideramos que éstos debían llegar a un consenso cercano al 70\% para calificar un ítem como válido.

En relación a la fiabilidad de las categorías construidas, establecimos que ésta se definiría a partir del grado de coincidencia entre diferentes jueces evaluadores, al asignar o confirmar segmentos en los códigos que componían las distintas categorías. En este proceso establecimos el grado de acuerdo a partir del uso del índice Kappa de Cohen (Cohen, 1960), adoptando como grado de acuerdo aceptable las coincidencias entre los evaluadores que alcanzaron o superaron el $75 \%$ en relación al total de segmentos codificados.

\section{RESULTADOS}

A continuación presentamos y discutimos los resultados, vinculándolos a los objetivos específicos que nos hemos propuesto conseguir.

\subsection{LOS COMPONENTES DE LA IDENTIDAD DOCENTE}

En relación a la identidad del docente, exponemos las categorías de análisis elaboradas dividas en tres ejes articuladores: concepciones vinculadas al rol docente y sobre enseñar y/o aprender en la universidad; concepciones en relación a metodologías o prácticas de enseñanza; y concepciones de contenido afectivo vinculadas con sus experiencias como profesores en la formación inicial docente.

Para este proceso de codificación hemos utilizado los datos extraídos de las 20 entrevistas iniciales (fase 1), los cuales nos permitieron elaborar una red de cuatro conjuntos temáticos. A continuación una tabla que resume las categorías y códigos elaborados con el propósito de analizar la identidad docente:

Tabla 2. Categorías de análisis de la identidad del docente universitario

\begin{tabular}{|ll|}
\hline \multicolumn{1}{|c|}{ Categorías } & \multicolumn{1}{c|}{ Códigos } \\
\hline Enseñar en la universidad & A.1 Conceptualizando la enseñanza \\
& A.2 Barreras en la docencia \\
A.3 Enseñar a futuros maestros \\
\hline Ser profesor universitario hoy & B.1 Hacerse docente universitario \\
B.2 El profesor "pasajero" \\
B.3 Roles en interacción-contradicción \\
\hline
\end{tabular}


Estudios Pedagógicos, vol. XL, Número Especial 1: 49-69, 2014

EL DESARROLLO DOCENTE DEL FORMADOR DE PROFESORES: UNA PROPUESTA ORIENTADA HACIA EL

ANÁLISIS DE INCIDENTES CRÍTICOS AUTÉNTICOS

\begin{tabular}{|ll|}
\hline Estrategias, metodologías y prácticas & C.1 Un modelo pedagógico propio \\
& C.2 Premisas para una buena clase \\
& C.3 Lo importante en la enseñanza \\
\hline Sensaciones y sentimientos en la docencia & D.1 El significado de ser docente \\
& D.2 Preocupaciones en la enseñanza \\
& D.3 Qué sienten al enseñar \\
\hline
\end{tabular}

Fuente. Elaboración Propia.

En lo que sigue, exponemos la definición de cada categoría incluyendo una cita como ejemplo de alguno de sus códigos constituyentes:

Categoría A. Enseñar en la universidad: esta categoría reúne las concepciones asociadas al proceso de enseñanza, sus objetivos, condiciones y características que permiten o dificultan orientar el proceso de aprendizaje. Particularmente, se hacen alusiones a la manera en que se entiende y se realiza la enseñanza en aulas de magisterio, y las complicaciones que se encuentran en dichos escenarios.

Ejemplo: “(...) un ingeniero por ejemplo no tiene por qué demostrarles a los otros como estudiantes que es ser un bueno profesor, en cambio que uno tiene que ser un buen profesor, y en ese sentido creo que hay un matiz didáctico, importante (...)”. Profesor/a 13, segmento 20, código: Enseñar a futuros maestros).

Categoría B. Ser profesor universitario hoy: esta categoría recoge representaciones en relación al trabajo cotidiano y los desafíos enfrentados por los profesores en la actualidad. Se puede identificar que el profesor de hoy se construye principalmente de manera autodidacta, desempeñando distintos roles, respondiendo a múltiples demandas y que se asume como un modelo de actitudes, conductas y de pensamiento.

Ejemplo: "uno en cierta medida se ve uno como estudiante y que no tuvo muy buenos, por decirlo no tuve muy buenos parámetro en los cuales pararme cuando fui formado como profesor, por lo general mis profesores de la universidad no eran muy comprometidos con la pedagogía... en el caso de mi carrera de origen, teníamos Geografía, teníamos Política, teníamos Historia, teníamos Educación, Sociología, entonces era mucho y cada uno disparaba para su lado, los sociólogos que fuéramos sociólogos, los psicólogos que seamos psicólogos, los pedagogos, pedagogos, entonces no había sintonía entre ellos(...)”. (Profesor/a 13, segmento 14, código: Hacerse docente universitario).

Categoría C. Estrategias, metodologías y prácticas: esta categoría agrupa códigos que incluyen representaciones sobre el quehacer en el aula, con el propósito de alcanzar los aprendizajes de los estudiantes, las expectativas sobre su desempeño y los propósitos que se deriven de su actuación. Estas representaciones involucran las maneras en que el profesor enseña, evalúa y gestiona conflictos, entre otras actuaciones.

Ejemplo: "Yo no tengo formación pedagógica, puedo saber que dice Paulo Freire y enseño el valor que tiene la educación en términos de socialización de las personas, pero no conozco un 
modelo educativo que te podría decir constructivista?, Montessori?, no, no los identifico ... tengo un modelo de referente a lo mejor autoconstruido de alguna forma porque lo que uno va entendiendo que es correcto, es deseable, que tiene que ver con de donde uno viene con tus propias experiencias como alumna y también con lo que uno se encuentra". (Profesor/a 10, segmento 69, código: Un modelo pedagógico propio).

Categoría D. Sensaciones y sentimientos en la docencia: esta categoría agrupa los códigos referidos a los sentimientos del profesor, las interpretaciones de sus emociones o sensaciones vinculadas al trabajo docente, representaciones afectivas que pueden ser de naturaleza positiva o negativa.

Ejemplo: "De impacto social, yo creo que eso es súper relevante porque tú como docente en el colegio tienes claro que hay un impacto y que tu trabajo es trascendente, pero cuando tú te instalas en la formación inicial docente la perspectiva es que efectivamente esa utopía de cambiar el mundo es posible, o sea efectivamente en pequeños cuotas, si es posible hacer cosas, claro ya no es un grupo de 10 alumnos como tengo yo aquí, sino que pueden ser 10, 12 alumnos, pero que van a tener 45 alumnos y el replicar todo eso, es como, como el sentido de trascendencia, el sentido del impacto de transformación social". (Profesor/a 5, segmento 18, código: El significado de la docencia universitaria).

En resumen, las categorías construidas en relación a la identidad docente nos revelan aspectos que se ponen en juego en la interacción con los estudiantes, y que tensionan ciertos posicionamientos del docente en cuanto resulta un desafío llegar a ser aquello que se ha internalizado como óptimo, para reflejarlo en el discurso y la acción. En este sentido, un aspecto particular que se expresa de varias formas es un posicionamiento identitario de ejemplo o patrón, en el cual tanto el mensaje y como la vía de representación se transforman en algo fundamental en la enseñanza dentro del contexto de la formación inicial docente.

Por último, debemos agregar que distinguimos otras particularidades en relación con la identidad, entre ellas el hecho de que los profesores se observan a sí mismos como potenciales transformadores de la realidad social, lo que pueden desarrollar en el contacto con los alumnos. Junto con lo anterior, otro aspecto que podemos inferir es el desarrollo de una identidad docente más vinculada a la experiencia y en una construcción estrechamente ligada a la práctica. En complemento, la emocionalidad o los sentimientos son un aspecto de la identidad docente que no emerge espontáneamente en el discurso de los participantes, con lo cual su integración o profundización resulta difícil, reflejando en parte una construcción identitaria más cercana a la racionalidad en la profesión.

\subsection{CARATERÍSTICAS DE LOS INCIDENTES CRÍTICOS}

Respecto de los resultados alcanzados en este tema, podemos señalar que dentro de los 62 segmentos seleccionados para el análisis hemos podido identificar una diversidad de IC, entre los que hay algunos que resultan significativos -en términos de su impacto-, y otros típicos, dada su recurrencia. Hemos logrado una caracterización, a partir de la elaboración de una categoría en la que se analizaron los relatos de los profesores participantes en la primera fase $(\mathrm{N}=20)$. A continuación, se presenta un resumen en la Tabla 3: 
Estudios Pedagógicos, vol. XL, Número Especial 1: 49-69, 2014

EL DESARROLLO DOCENTE DEL FORMADOR DE PROFESORES: UNA PROPUESTA ORIENTADA HACIA EL

ANÁLISIS DE INCIDENTES CRÍTICOS AUTÉNTICOS

Tabla 3. Categoría de análisis para incidentes críticos primera fase

\begin{tabular}{|ll|}
\hline \multicolumn{2}{|c|}{ CATEGORÍA: LOS INCIDENTES CRÍTICOS } \\
\hline \multicolumn{1}{|c|}{ Código } & \multicolumn{1}{c|}{ Definición } \\
\hline $\begin{array}{l}\text { Explicando los incidentes críticos } \\
\text { (26 segmentos) }\end{array}$ & $\begin{array}{l}\text { Aborda hipótesis, fuentes o variables que los profesores } \\
\text { consideran que intervienen en la ocurrencia de un IC. } \\
\text { Esto puede incluir pensamientos inmediatos, } \\
\text { interpretaciones posteriores, generalizaciones }\end{array}$ \\
\hline $\begin{array}{l}\text { Consecuencias de un incidente crítico } \\
\text { (13 segmentos) }\end{array}$ & $\begin{array}{l}\text { Secuelas o efectos que se derivan o suponen a partir de la } \\
\text { ocurrencia de un IC. Esto puede incluir cambios en la } \\
\text { planificación en la relación con estudiantes, en actitudes } \\
\text { del profesor, entre otros }\end{array}$ \\
\hline $\begin{array}{l}\text { Reacción ante los incidentes críticos } \\
\text { (23 segmentos })\end{array}$ & $\begin{array}{l}\text { Formas de enfrentamiento, evitación o respuesta dada al } \\
\text { momento en que el profesor vivencia o interpreta un IC }\end{array}$ \\
\hline
\end{tabular}

Fuente. Elaboración Propia.

A partir de los segmentos analizados y en base a la categoría presentada, podemos establecer que un IC puede ocurrirle a todo profesor, cualquier día de clase o con cualquier grupo de estudiantes. Para ello, bastará, por ejemplo, que uno o varios de los miembros del aula se resistan a seguir instrucciones, que los estudiantes encuentren una distracción que los aleje de la tarea de aprendizaje, o no encuentren sentido a la actividad de evaluación que el docente ha planificado, todos ellos hechos que pueden darse en las aulas universitarias. Cabe agregar que esta primera aproximación y caracterización nos permitió contar con un plano conceptual emergente, que pudo ser utilizado como marco comprensivo general en las actividades de formación (Fase 2).

En seguida, la figura presentada grafica algunas de las preguntas y respuestas que podemos formularnos en torno a los IC y hacia las cuales, en parte, orientamos este estudio (Figura 2):

Figura 2. Esquema para una caracterización de los IC

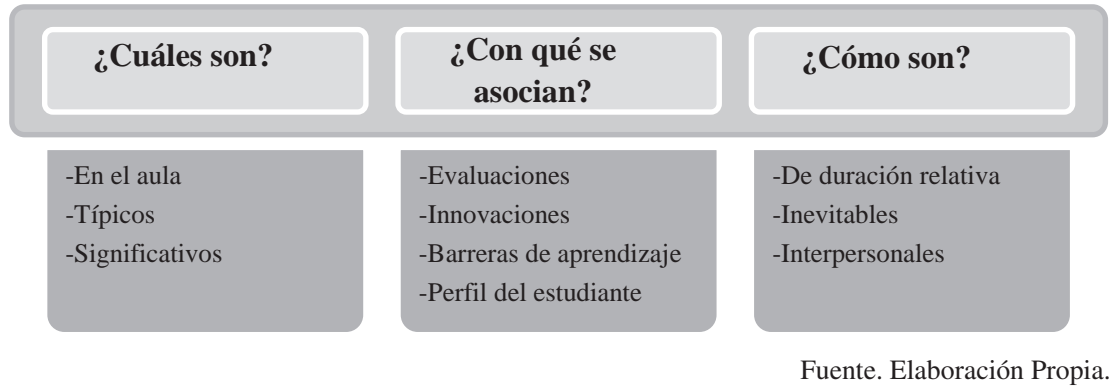

A partir de estos resultados, igualmente podemos acordar que existen múltiples variables que confluyen en el origen de un IC, tales como escenarios o condiciones que intervienen en situaciones de crisis y que facilitan su ocurrencia en el aula, entre las que por ejemplo se encontrarían las innovaciones o las situaciones de evaluación, entre otros. 


\subsection{EL PROGRAMA DE FORMACIÓN BASADO EN INCIDENTES CRÍTICOS}

En una primera aproximación a la formación permanente del profesorado universitario y con el propósito de obtener resultados que nos permitieran hablar de un estado inicial, hemos desarrollado un proceso de análisis utilizando los datos extraídos en la Fase 1, lo cual nos permitió elaborar lo siguiente (Tabla 4):

Tabla 4. Categorías de análisis para la formación docente

a) La formación y aprendizaje profesional del profesorado universitario

a1. La institución como espacio de contradicciones

a2. Necesidades de formación y encuentro

Fuente. Elaboración Propia.

Tal como podemos observar, en esta categoría recogemos representaciones que caracterizan los entornos institucionales, haciendo referencias a las barreras que entorpecen el desarrollo profesional o las condiciones para una formación docente óptima y/o ajustada. Lo anterior se vincula a interpretaciones de la realidad institucional, exigencias, condiciones o prioridades, entre otros aspectos relacionados. A continuación definimos los dos códigos constituyentes de esta categoría y los acompañamos de un ejemplo representativo.

a1. La institución como espacio de contradicciones. Este código recopila representaciones sobre las instituciones. Ellas son visualizadas como escenarios profesionales más bien individualistas, poco abiertos a la innovación y colaboración entre colegas, lo cual puede ser visto como una contradicción, considerando el rol social atribuido a la universidad (17 segmentos, 9 docentes).

Ejemplo: “... Oportunidades hay, lo que no hay es un diseño de, no hay un camino, o sea en el fondo no son oportunidades institucionalizadas, no es que haya un programa de formación, o sea algo que sea atractivo, que sea dialógico, que sea contextual, y que esté al día con lo que esté sucediendo a nivel mundial, no, eso no hay, lo que hay es la oportunidad para que el que está atento, lo busca de acuerdo a sus intereses". (Profesor/a 19, segmento 107).

a2. Las necesidades de formación y encuentro. Este código reúne representaciones acerca de las necesidades de formación del profesorado universitario y la importancia de tener espacios de encuentro con pares. Igualmente, se rescata una visualización de las posibilidades que abriría un aprendizaje colectivo y una formación desarrollada a partir de necesidades del entorno inmediato (39 segmentos, 12 docentes).

Ejemplo: "Algo más colegiado si, de repente uno como muy sola tratando de resolver los conflictos, a lo mejor uno no lo hace ni bien, ni mal, sino que es parte de, incluso de las misma relaciones que uno establece, pero sería creo yo una condicionante, creo una condición importante, ahora que lo estoy mirando, una de las cosas que yo observo en la universidad es el poco trabajo entre los profesores, y esa sí que para mí sería una condición para sentirme más seguramente más preparada frente a los distintos grupos". (Profesor/a 5, segmento 123). 
De esta manera, y a partir de los códigos elaborados, podemos señalar que los profesores identifican ciertas necesidades y manifiestan con claridad algunas características de la formación que requieren, pues son ellos los que reconocen habilidades o dificultades cuando enfrentan problemas como conflictos emocionales, barreras en el aprendizaje de sus estudiantes, etc. Por otro lado, la percepción de la formación como un camino en solitario y en donde la autogestión es la vía de solución, también es digno de análisis, dado que nos situamos dentro de instituciones de formación en donde el mejoramiento de la docencia debiera ser considerado explícitamente, al mismo tiempo que otorgar los medios para ello.

Desde la perspectiva de este estudio y bajo la premisa de que los programas de desarrollo deben responder a las necesidades y problemas reales que enfrentan los profesores, organizamos la segunda fase de la investigación tomando en cuenta la inmersión en el contexto y el establecimiento de vínculos de confianza, como parte de la esfera relacional en la intervención. A partir de lo anterior, planteamos un programa desde dos miradas de la formación docente: a) desde un enfoque auténtico, en tanto consideramos el aprendizaje como un proceso que incorpora la experiencia y se sitúa en un contexto determinado, para lo cual recogimos datos desde la cotidianeidad; y b) desde una visión de la formación, donde el mediador es un par que cumple un rol de asesor psicoeducativo y que contribuye con orientaciones para la transformación docente; y que atiende a las necesidades desde la pertinencia y la inmersión en la realidad estudiada. Para lograr esto, realizamos entrevistas previas y posteriores a la formación en grupo, así como formaciones individuales con cada uno de los docentes, de manera de ir respondiendo a ciertas necesidades de manera contextualizada. En relación a las características del programa, podemos señalar que éste se desarrolló a lo largo de seis sesiones de manera quincenal, en un tiempo aproximado de 3 meses.

A continuación la Tabla 5 resume los temas trabajados en las sesiones, así como la modalidad y el número de asistentes:

Tabla 5. Resumen del programa de formación

\begin{tabular}{|lccc|}
\hline Sesión & Tema & Formato & Participantes \\
\hline Primera & Desafíos del docente & Grupal & 6 \\
\hline Segunda & Incidentes en el aula & Individual & 5 \\
\hline Tercera & Análisis de un caso (1) & Grupal & 5 \\
\hline Cuarta & Cómo enfrentamos un IC & Individual & 5 \\
\hline Quinta & Análisis de un caso (2) & Individual & 4 \\
\hline Sexta & Un caso real compartido (3) & Grupal & 5 \\
\hline
\end{tabular}

Fuente. Elaboración Propia.

En relación al papel que jugaron los IC en la formación, debemos señalar que como dispositivo fueron utilizados a partir de tres fuentes: la primera, con ejemplos cercanos a la realidad, pero ficticios; la segunda fruto de una investigación anterior en el contexto nacional en la que se obtuvo ejemplos cercanos a los participantes; y tercero, como producto del mismo estudio y de los cuales los participantes eran protagonistas, lográndose progresivamente una mayor de autenticidad. 


\subsection{LA VALORACIÓN DEL PROGRAMA DE FORMACIÓN BASADO EN INCIDENTES CRÍTICOS}

En relación a los resultados de la formación, seleccionamos para el análisis exclusivamente segmentos de la entrevista de evaluación final, realizada en un plazo de entre 2 y 3 meses luego de finalizado el programa de formación. A continuación presentamos una breve descripción de cada docente participante, seguida de una selección de segmentos relevantes en relación a su evaluación del proceso, en consideración a las transformaciones que perseguía la formación realizada.

Caso 1. Este caso es un profesor de nacionalidad extranjera que realiza clases en pregrado y postgrado, con una trayectoria docente menor a los 10 años y que en su actual universidad lleva trabajando menos de 3 años. Durante la formación, este profesor se mostró muy crítico de las competencias y el perfil que observa en sus estudiantes, así como también sobre el trato y las condiciones establecidas por parte de la universidad para el rendimiento académico de los futuros profesores. Desde un principio planteó su disponibilidad a trabajar y se mostró abierto a exponer sus inquietudes, sin embargo, al inicio de la formación no reconocía la ocurrencia de IC en su experiencia, lo cual fue evolucionando en el tiempo. A continuación una cita en relación a su experiencia dentro del proceso formativo:

“(...) en algún momento te hablé de eso, no recuerdo muy bien lo que te dije, yo creo que así como que esto me haya servido para tener un proceso de aprendizaje yo creo que no, lo que sí conceptualizar una realidad siempre es enriquecedor, entonces claro una realidad que a lo mejor antes de participar en este trabajo únicamente era una vivencia a la cual yo no le había puesto nombre y como no le había puesto nombre probablemente no hubiera generado mucha reflexión al respecto, ahora se enriquece, ahora se enriquece (...)". (Segmento 51, entrevista final).

Por otra parte, tal como en otros relatos, dentro de la entrevista de evaluación final vuelven a emerger críticas hacia la formación permanente del profesorado universitario y las contradicciones enfrentadas en la universidad. Si bien estas contradicciones se relacionan indirectamente con los indicios de cambio en la identidad, sí reflejan en parte un malestar que se mantiene latente en relación a los roles de enseñante e investigador, conflicto que es identificado y explicitado claramente:

"La universidad no atiende nada de tu proceso de aprendizaje, de hecho no tienen ni idea de cómo uno está aprendiendo, no tienen idea, entonces las universidades en general, y esta en particular, no están preocupadas de tu aprendizaje, vamos, yo ni siquiera lo he leído, a lo mejor lo dicen en algún sitio, pero ni siquiera me asusta, ellos están preocupados de otras cosas, asumen que las cosas por las que están ocupados te permiten seguir aprendiendo, pero eso no es cierto, ¿ah?, ellos están preocupados de mayor productividad científica asumiendo que cuando tú publicas más aprendes más...”. (Segmento 65, entrevista final).

“... para mí fue enriquecedor, más enriquecedoras las conversaciones contigo que las del grupo, pero tampoco pude asistir a muchas, y el grupo me sirvió para reafirmar ciertas cosas, que los profesores tenemos miedo a que nos miren, que los profesores tenemos miedo a que nos digan y que cuesta mucho trabajo cambiar, eso ya lo sabía, pero el grupo me permite de alguna manera reafirmarlo. (Segmento 67, entrevista final).

Finalmente, en relación a este docente recogemos una cita que nos da indicios de un 
Estudios Pedagógicos, vol. XL, Número Especial 1: 49-69, 2014

EL DESARROLLO DOCENTE DEL FORMADOR DE PROFESORES: UNA PROPUESTA ORIENTADA HACIA EL

ANÁLISIS DE INCIDENTES CRÍTICOS AUTÉNTICOS

cambio fundamental, y es la visión del otro que ya no resulta una amenaza dentro del aula, en donde puede compartirse.

“(...) yo ya había tenido experiencia de gente que había ido a observarme pero, habían sido experiencias muy puntuales y de gente con la cual tenía mucha confianza y mucha amistad, entonces ahí el proceso es diferente, entonces cuando tú me dices de venir a una clase y yo acepto y yo me siento bien, en ningún momento me siento incómodo, en ningún momento siento que me están evaluando, aunque a lo mejor sí me estás evaluando. (Segmento 69, entrevista final).

En relación a esto último, nos atrevemos a decir que esta visión incorpora un cambio en la conceptualización de una evaluación entre pares más enjuiciadora, hacia una visión de colaboración entre pares.

Caso 2. Este caso es una profesional de nacionalidad extranjera que imparte docencia en pregrado y postgrado, con una experiencia en la docencia menor a 10 años, siempre ha trabajado en la misma universidad, aunque en paralelo también ha trabajado en otras instituciones privadas. Esta profesora, aunque al inicio manifestó su acuerdo de videograbar las clases, durante el proceso esta decisión cambió. En general, intervino regularmente en las sesiones y se justificó al ausentarse en la segunda sesión.

Dentro de la entrevista de evaluación, realiza un análisis y formula una nueva conceptualización personal de los incidentes, manifestando lo siguiente:

"Yo creo que en términos generales siempre hay incidentes críticos cuando se llega a los límites de la normalidad, como en cualquier sociedad, cuando llegaste al límite de lo que la norma permite, en el sentido de que claro las excepciones generan (...) generalmente hay incidentes que tienen que ver con salirse de eso, de la normalidad, de seguirle el ritmo a la clase, de respetar la autoridad del profesor, las notas, cuando se desestructura (...) hay más que eso, y ahí sigo, nuevamente el incidente crítico pasa a ser un pequeño grano frente a una situación mayor aún (...)”. (Segmento 60, entrevista final).

En resumen, a partir de los segmentos de discurso anteriores, podemos encontrar de manera predominante indicios de cambio a nivel conceptual en relación a los incidentes críticos. Por otro lado, en vinculación a lo anterior, encontramos nuevamente una evaluación de la cultura docente en la universidad como un espacio que se reduce al actuar individual:

"lo frecuente que sucede, y que tiene que ver, y yo creo que la sorpresa tiene que ver con un porque en nuestras universidades tampoco generan, ya lo conversamos alguna vez, espacios o momentos donde esto pueda ser discutido, en un trabajo con otros, de tipo trabajo en par, acá cada cual va a lo suyo (...)”. (Segmento 60, entrevista final).

La manifestación de este tipo de juicios nos parece relevante en la medida que explicita ciertos elementos de la cultura, pero también como un factor que desafió constantemente la realización del presente estudio.

Caso 3. Se trata de un profesional que realiza clases en pregrado, con una trayectoria docente menor a 3 años, la única universidad en la que ha trabajado es en la que se encuentra actualmente. Desarrolla docencia en la Facultad de Educación, aunque su especialidad y adscripción institucional es en otra área. 
Desde un principio, este profesor se presentó muy abierto a compartir experiencias y reflexiones, su disponibilidad a trabajar era plena y se mostró muy interesado en compartir espacios fuera de las sesiones planificadas. En general, durante las sesiones fue muy crítico del trato y perfil que observa en sus estudiantes de pedagogía, sobre todo en lo referido al nivel de exigencia que debería esperarse, más cuando comparaba este perfil con el de estudiantes de otra facultad a los que realiza clases. En el transcurso de su participación, este docente se fue haciendo más consciente de que al desarrollar docencia en la FID, tanto el contenido como la forma en que es comunicado tienen suma importancia, fue así como un matiz metodológico fue impregnando su discurso. Al respecto nos señaló: “ (...) lo otro que me he dado cuenta últimamente es que debe tener mucho conocimiento acerca de cómo impartir una clase, de cómo hacer docencia digamos" (Segmento 7, entrevista final).

En relación a otros indicios de cambio, encontramos alusiones directas a los aprendizajes de este docente, a continuación un par de segmentos de relato que explicitan dichos aprendizajes:

"Creo que dentro de las cosas que aprendí de alguna manera estrategias de cómo responder ante algunos incidentes críticos, lo habíamos conversado durante las reuniones, que todos los docentes habíamos tenido algunos incidentes críticos y la propia experiencia hace que uno vaya respondiendo distinto y que de cierta manera ya no sean incidentes críticos los que eran ayer, yo creo que me ha dado un poco de experiencia, y lo otro de ponerme a pensar de cuáles son los incidentes críticos que me han ido ocurriendo, y cómo tendría que reaccionar en las siguientes ocasiones (...)”. (Segmento 37, entrevista final).

"No, ahora trato de incorporarlos (los incidentes críticos), es difícil incorporar el incidente como para proponer algo nuevo en la clase, pero trato de incorporar a los sujetos con que tengo el incidente, de alguna manera, en la clase, para que se sientan comprometidos con lo que estamos haciendo". (Segmento 45, entrevista final).

Finalmente, en relación a los incidentes críticos, pudimos identificar evidencias de cambio, y como bien podemos apreciar en estos relatos, hay distinciones tanto en la conceptualización como en las estrategias para su utilización. Ambos elementos nos indican una trasformación más profunda en relación con la identidad de este docente.

Caso 4. Se trata de una profesora que imparte docencia en el pregrado, con una experiencia docente menor a 10 años, de los cuales la mayoría ha estado trabajando en la misma universidad. Desde el inicio, esta profesora reconoció en su experiencia incidentes críticos, lo cual facilitó que compartiera algunos episodios vivenciados, así como sus reflexiones e inquietudes en torno a ellos. Esta docente asistió a todas las sesiones y, por iniciativa propia, fuera de lo planificado, acudió un par de veces en búsqueda de asesoramiento en relación a su clase. A continuación exponemos un segmento de relato en el cual la docente reconoce un cambio en su vivencia de los incidentes críticos:

"Cuando llegaste hablando de los incidentes críticos en la primera encuesta, los primeros correos, la verdad es que no estaba muy involucrada, hasta que se vivió, por eso te hablo de la vivencia, desde la práctica (ríe), tú fuiste testigo de uno de los incidentes, que de repente pasan muchos más, y cuando compartimos con los demás colegas también me quedó mucho más claro, qué se considera como incidente crítico, a pesar de que no manejo la conceptualización teórica que tú tienes, pero sí entiendo que, y hoy día soy capaz de decir que cuando se genera un 
Estudios Pedagógicos, vol. XL, Número Especial 1: 49-69, 2014

EL DESARROLLO DOCENTE DEL FORMADOR DE PROFESORES: UNA PROPUESTA ORIENTADA HACIA EL

ANÁLISIS DE INCIDENTES CRÍTICOS AUTÉNTICOS

problema me cuestiono otra vez para cambiar algunas estrategias, entonces encontré súper valiosa la investigación que tu realizabas para hacerte un poco más consciente de que esas tensiones, esas problemáticas, esos incidentes en el aula, ahora, sí le sirven a los estudiantes mejor, aprovecharlos, reciclar". (Segmento 73, entrevista final).

Pese a la dificultad que implica el reconocimiento de ciertos elementos afectivos en la identidad, a continuación presentamos un par de citas que insinúan un cambio en relación a al significado y utilización del incidente en el aula:

“(...) porque siempre yo me reía contigo, porque cuando estaba pasando algo malo, malo, digamos incómodo para los estudiantes, incómodo para mí, y se me aparecía la Claudia, entonces (risas) pero tenía la imagen ¿cachai?, tenía la imagen, pensaba "aquí hay un incidente crítico (risas), "analicemos", entonces claro, hace un tiempo atrás "se desconfigura todo, todos peleando, todo mal" y ahora no, claro también se pone cierto freno(...), no sé si lo hubiera hecho hace un año atrás". (Segmento 99, entrevista final).

Tal como podemos apreciar, hay un cambio en la concepción del incidente, esto con repercusiones en las estrategias que puedan implementarse, así como en la emocionalidad experimentada, amplificando un impacto en la identidad de la docente.

Caso 5. Este caso es de un profesor que realiza clases en pregrado, con una trayectoria docente menor a 10 años, de los cuales 2 ha trabajado en la universidad donde se encuentra actualmente. Desde el principio, este profesor se presentó disponible a compartir vivencias, sin embargo en los primeros encuentros manifestó que era difícil para él reconocer incidentes críticos en su experiencia, por su forma de enfrentar y comprender la docencia. Se ausentó sólo en la quinta sesión, debido a un viaje.

Uno de los aspectos que más llama la atención es que al realizar la evaluación con este docente, él valora los IC de una manera particular y los vincula con otras propuestas en relación a su disciplina, a continuación lo que nos ha señalado:

"Es súper sencillo(dibuja en una hoja), imagínate tres círculos así como el logo de pinturas "ceresita", entonces este tipo le llama a la zona del centro, la zona de confort, situaciones donde se tienen estímulos a las que el sujeto ya tiene respuesta, después al círculo de en medio le llama zona de aprendizaje, situaciones que generan estímulos para los cuales la persona no tiene respuesta, pero está dentro de sus capacidades generarlas, y el tercer círculo que está fuera le llama zona de pánico, demanda de respuesta que está fuera del alcance de las posibilidades de la persona en general, entonces en algún minuto me hizo el click el cruce entre todo lo que tú estabas proponiendo, trabajando, conceptualizando como incidente crítico, pero lo vi a la luz de lo que este autor describía como zona de pánico”. (Segmento 51, evaluación final).

Por otra parte, este profesor realiza una crítica que tiene mucha relación con las dificultades enfrentadas durante la fase de intervención. A continuación lo que nos ha relatado en la última entrevista:

"Bueno metodológicamente creo que no se me ocurre nada para complementar, era más que nada la constitución del grupo(...), metodológicamente descartar de plano todo lo que significa completación de cuestionarios fuera de los tiempos de encuentro, se hacía muy complejo 
destinarle tiempo al proceso más allá de las reuniones, y lo otro, quizás más estratégico, yo valoro mucho, mucho lo que tú hiciste, pero hacerlo como estudiante de postgrado, en base a la buena onda de la gente, es súper tortuoso, tendría mucho sentido como un, nos molestan tanto en el (Departamento encargado de la calidad docente) acá para cuestiones que claramente no son tan, me refiero por ahí, podría entrar como un programa institucional, y tener otro respaldo, ese sería un paso alterno, los resultados de tu trabajo darían para avanzar en ese sentido". (Segmento 61, evaluación final).

Finalmente, al contrario de lo que podría señalarse, esta crítica reconoce en la formación su utilidad, y por tanto una valoración al potencial impacto de mayor envergadura si se desarrollará en un contexto de mayor formalidad y con un apoyo institucional.

\section{CONCLUSIONES}

En respuesta a los objetivos que nos planteamos en esta investigación, pasamos a describir las principales conclusiones derivadas de este estudio:

Podemos afirmar que la identidad del docente universitario de magisterio está fuertemente influenciada por la relación entre el contenido y la vía en que éste es desarrollado en el aula. Ello se refleja en una auto-representación del docente como un ejemplo vivo de la teoría y la práctica pedagógica, pero al mismo tiempo como un profesional creador de espacios de aprendizaje, con lo cual el mensaje y su formato cobran mucha importancia. Esto nos permite pensar, además, en evidencias sobre una identidad docente en proceso, que no es anulada o sometida por un rol de experto disciplinar.

Los sentimientos como dimensión en la identidad docente emergen débilmente y de manera ocasional en el discurso del profesorado. Éste es un aspecto que deberíamos problematizar, dado que es reconocida la importancia de la dimensión afectiva en situaciones de cambio educativo, satisfacción laboral y motivación hacia el trabajo, y su influencia las decisiones de los docentes en sus actividades de enseñanza.

Podemos afirmar que surgen fuertemente tres roles docentes: enseñante, investigador y gestor. Los primeros dos son ampliamente reconocidos y valorados por los docentes, aun cuando hay políticas o factores institucionales que condicionan su desarrollo. Diferente es la valoración del rol de gestor, que es relegada a un lugar secundario y considerada poco relevante para el profesorado. En relación a los dos roles más fuertemente referidos -investigador y enseñante- observamos cierto grado de distancia aun cuando los profesores consideran estos ámbitos como complementarios y que son parte de un desarrollo profesional a integrar. Sin embargo, esta integración se visualiza como un desafío ya que muchas veces la universidad muestra un discurso contradictorio.

Los IC relatados por los docentes se asocian a tres situaciones principalmente, las evaluaciones del aprendizaje, déficits en las competencias de los estudiantes y la introducción de cambios en las normas o metodologías de aula. Estos incidentes emergen mayoritariamente como episodios únicos, no obstante pueden presentar consecuencias posteriores en la relación con los estudiantes y en las metodologías o procedimientos seguidos en la clase.

Los incidentes críticos son considerados como un valioso recurso para el diseño de propuestas de formación pertinentes y ajustadas. En este sentido se valora el espacio de formación desarrollado, sobre todo, desde la perspectiva de un trabajo colegiado y las 
Estudios Pedagógicos, vol. XL, Número Especial 1: 49-69, 2014

EL DESARROLLO DOCENTE DEL FORMADOR DE PROFESORES: UNA PROPUESTA ORIENTADA HACIA EL

ANÁLISIS DE INCIDENTES CRÍTICOS AUTÉNTICOS

posibilidades que ofrece el asesoramiento en un contexto colaborativo. En esta línea, los docentes, a pesar de la alta demanda de sus funciones o actividades académicas, evalúan positivamente el poder contar con un tiempo para la reflexión, resolver las preocupaciones asociadas la calidad de su enseñanza y aquellas derivadas de las relaciones con su alumnado.

Entre los profesores participantes en la formación, identificamos un cambio en la concepción de IC. Este cambio se vincula con una visión del IC asociado a un sistema externo de representación, que permite explicitar concepciones propias, compartirlas, discutirlas y, al mismo tiempo, problematizar situaciones para una búsqueda de soluciones. Asimismo, los docentes se distancian del IC como un hecho meramente negativo y desestabilizante, rescatándose su utilidad para la formación y el análisis reflexivo.

Los resultados de nuestro estudio sugieren que la formación permanente del profesorado universitario aún es un tema pendiente en Chile en la medida en que es vista como una responsabilidad individual, solitaria y distante de lo que significa ser docente en la actualidad, y que desde el punto de vista de los incentivos y prioridades institucionales es un ámbito de reciente preocupación, explicitación y desarrollo.

\section{REFERENCIAS BIBLIOGRÁFICAS}

Akkerman, S., \& Meijer, P. (2010). A dialogical approach to conceptualizing teacher identity. Teacher and teaching education, vol.30, 1-12.

Badia, A., Monereo, C. y Meneses, J. (2011). El profesor universitario: identidad profesional, concepciones y sentimientos sobre la enseñanza. En Román, J., Carbonero, M. y Valdivieso, J. Educación, aprendizaje y desarrollo en una sociedad multicultural (pp. 5647-5661). Valladolid: Asociación Nacional de Psicología y Educación.

Badley, G. (2000). Developing Globally-Competent University Teachers. Innovations in Education \& Training International, vol.37, n.3, 244-253.

Briggs, S. (2005). Changing roles and competencies of academics. Active learning in higher education, vol.6, n.3, 256-268.

Cohen, J. (1960). A coefficient of agreement for nominal scales. Educational and Psychological measurement, vol.20, n.1, 37-46.

Contreras, C., Monereo, C. y Badia, A. (2010). Explorando en la identidad: ¿Cómo enfrentan los docentes universitarios los incidentes críticos que ocurren en las aulas de formación de futuros profesores? Estudios pedagógicos, vol.36, n.2, 63-81.

Cornejo, J. (2007). La formación de los formadores de profesores ¿Para cuándo en Chile? Revista Pensamiento educativo, vol.41, n.2, 37-55.

Day, C. (2005). Formar docentes. Cómo, cuándo y en qué condiciones aprende el profesorado. Madrid: Narcea.

Day, C. (2006). Pasión por enseñar. La identidad personal y profesional del docente y sus valores. Madrid: Narcea.

De la Cruz, M. (2003). Necesidad y objetivos de la formación pedagógica del profesor universitario. Revista de educación, n.331, 35-66.

Galaz, A. (2011). El profesor y su identidad profesional ¿facilitadores u obstáculos del cambio educativo? Estudios pedagógicos, vol.37, n.2, 89-107.

Guskey, T.R. (2000). Evaluating professional development. Thousand Oaks, California: Corwin Press.

Hargreaves, A. (2005). Profesorado, cultura y postmodernidad. Cambian los tiempos, cambia el profesorado ( $\left.5^{\mathrm{a}} \mathrm{ed}.\right)$. Madrid: Morata. 
Hernández, R., Fernández, C. y Baptista, P. (2010). Metodología de la investigación (5ª ed.). México D.F.: McGraw-Hill.

Karmiloff-Smith, A. (1994). Más allá de la modularidad: La ciencia cognitiva desde la perspectiva del desarrollo. Madrid: Alianza.

Meijers, F. (2003). Career learning in a changing world: The role of emotions. International journal for the advancement of counselling, vol.24, n.3, 149-167.

Monereo, C., Badia, A., Bilbao, G., Cerrato, M. y Weise, C. (2009). Ser un docente estratégico, cuando cambiar la estrategia no basta. Cultura y Educación, vol.21, n.3, 237-256.

Monereo, C. y Badia A. (2011). Los heterónimos del docente. Identidad, selfs y enseñanza. En C. Monereo y J. Pozo (Eds.), La identidad en Psicología de la Educación. Necesidad, utilidad y límites (pp. 57-76). Madrid: Narcea.

Monereo, C. y Monte, M. (2011). Docentes en tránsito. Incidentes críticos en Educación Secundaria. Barcelona: Graó.

Navarro, Y., López, M., Climent, J. y Ruíz, M. (2012). Desarrollo de competencias de gestión de conflictos en la formación universitaria. Revista Iberoamericana de Educación, n.60, 2.

Pozo, J. (2006). La nueva cultura del aprendizaje en la sociedad del conocimiento. En J. Pozo, N. Scheuer, M. Pérez, M. Mateos, E. Martin y M. De la Cruz (Coords.), Nuevas formas de pensar la enseñanza y el aprendizaje. Las concepciones de profesores y alumnos (pp. 29-53). Barcelona: Graó.

Strauss, A. y Corbin, J. (2002). Bases de la Investigación cualitativa: Técnicas y procedimientos para desarrollar la Teoría fundamentada. Medellín: Editorial Universidad de Antioquia.

Tedesco, J. y Tenti, E. (2002). Nuevos tiempos, nuevos docentes. Buenos Aires: Instituto Internacional de planeamiento de la educación-UNESCO.

Tripp, D. (1993). Critical incidents in teaching. Developing professional judgment. Londres: Routledge.

Zabalza, M. (2009). Ser profesor universitario hoy. La cuestión universitaria, n.5, 69-81. 
